\title{
Sleep problems among adolescents within child and adolescent mental health services. An epidemiological study with registry linkage
}

\author{
Mari Hysing ${ }^{1,2}(\cdot) \cdot$ Ove Heradstveit $^{2,3} \cdot$ Allison G. Harvey $^{7} \cdot$ Sondre Aasen Nilsen ${ }^{2} \cdot$ Tormod Bøe $^{1,2} \cdot$ Børge Sivertsen $^{4,5,6}$
}

Received: 3 August 2020 / Accepted: 25 October 2020 / Published online: 7 November 2020

(c) The Author(s) 2020

\begin{abstract}
Sleep problems are prevalent among adolescents, especially among those diagnosed with mental health disorders. There is insufficient knowledge about sleep among adolescents within child and adolescent mental health services (CAMHS) in comparison to the general population. The data are drawn from the youth@ hordaland study, a large population-based study conducted in 2012, linked to the Norwegian Patient Registry (NPR) $(n=9077)$. Psychiatric disorders were based on clinical diagnoses from the NPR, while insomnia, delayed sleep-wake-phase disorder (DSWPD), and other sleep problems/patterns were assessed by self-report questionnaires from youth@ hordaland. The prevalence of diagnosed sleep disorders among adolescents seeking mental health services was $0.6 \%$, yielding an estimated prevalence of $0.07 \%$ of the population. However, questionnaire-based measurement of insomnia from the youth@ hordaland study indicated that insomnia was highly prevalent across disorders in comparison to a reference group of adolescents who were not within mental health care. Insomnia ranged from 29\% among adolescents diagnosed with ADHD ( $\mathrm{PR}=1.79 ; 95 \%$ CI 1.41-2.29) to 48\% among adolescents diagnosed with depression ( $\mathrm{PR}=2.53,95 \%$ CI 2.19-2.92). All diagnostic groups had a mean sleep efficiency below (85\%), indicating poor sleep quality. Insomnia, delayed sleep-phase wake disorder, and poor sleep efficiency were confirmed as transdiagnostic sleep problems across psychiatric disorders. In addition, some disorder-specific patterns emerged, such as a higher prevalence of insomnia among adolescents with depression, and DSWPS among adolescents with conduct disorder. This underscores the need for treating sleep problems in CAMHS, and transdiagnostic treatment approaches are warranted.
\end{abstract}

Keywords Insomnia $\cdot$ Short sleep duration $\cdot$ DSWPD $\cdot$ Psychiatric disorders $\cdot$ Adolescence

\section{Introduction}

Mari Hysing

Mari.hysing@uib.no

1 Department of Psychosocial Science, Faculty of Psychology, University of Bergen, Bergen, Norway

2 Regional Centre for Child and Youth Mental Health and Child Welfare, NORCE Norwegian Research Centre, Bergen, Norway

3 Center for Alcohol \& Drug Research, Stavanger University Hospital, Stavanger, Norway

4 Department of Health Promotion, Norwegian Institute of Public Health, Bergen, Norway

5 Department of Research \& Innovation, Helse-Fonna HF, Haugesund, Norway

6 Department of Mental Health, Norwegian University of Science and Technology, Trondheim, Norway

7 Department of Psychology, University of California, Berkeley, USA
Sleep problems are prevalent among adolescents after puberty [1]. Sleep during this developmental stage is characterized by short sleep duration on weekdays, a delayed sleep phase, and a high prevalence of insomnia [2,3]. Adolescents with mental health problems have an even higher prevalence of sleep problems across diagnosed disorders [4].

Sleep problems are commonly listed as core symptoms of many psychiatric disorders, including major depressive disorder (MDD) and generalized anxiety disorder (GAD) [5]. Sleep problems may be a causal factor in the development of such psychiatric disorders [6], but also a consequence of these [7]. This bidirectional perspective is in sharp contrast to the previously often held belief that sleep problems were secondary to a psychiatric disorder [8], and thus rarely diagnosed or targeted in mental health care interventions. This change in perspective is also manifested in the transition from DSM-IV to DSM-5, where the 
previous distinction between insomnia with and without a co-existing mental disorder is no longer included in the latest revision $[5,9,10]$. This shift has also led to a greater emphasis on diagnosing and treating sleep problems when these co-occur alongside a mental health disorder.

Still, we know little about the prevalence of sleep problems among adolescents seeking help from child and adolescent mental health services (CAMHS). One study that has shed light on sleep patterns among adolescents with psychiatric disorders, is the National Comorbidity Study from 2000 to 2004 that is based on a nationally representative sample recruited from the USA. This study assessed sleep patterns, not sleep disorders, across several psychiatric diagnoses among over 10,000 adolescents aged 13 to 18 years. The results indicated later weeknight bedtime, shorter weeknight sleep duration, greater weekend bedtime delay, and both short and long periods of weekend oversleep were associated with increased odds of mood, anxiety, substance use, and behavioral disorders [4]. A Norwegian study found a higher level of sleep problems assessed by five items from the Youth Self Report Questionnaire in the clinical sample of adolescents attending specialty health care clinic (31\%), relative to a community sample (5\%) [11]. The importance of increasing our attention to sleep problems in mental health care services in general was also demonstrated in a study of adults receiving treatment in public mental health service clinics, where sleep problems were an independent predictor of impaired functioning and fewer benefits from treatment [12].

Sleep problems co-occurring with psychiatric disorders may take many forms including insomnia [7, 13, 14], circadian rhythm disorders, and a short sleep duration [15]. Although sleep problems can be considered a transdiagnostic phenomenon [16], the prevalence and patterns of sleep problems may vary across different psychiatric disorders. Employing a unique linkage approach whereby official data from registries that record mental illness based on clinician diagnoses, provides opportunity to compare adolescents connected to mental health care versus adolescents not connected to care. The linkage also allows for similar detailed sleep assessment for both groups. Sleep patterns and sleep problems differ by gender [1,3], and similarly there are gender differences in the prevalence of psychiatric disorders [17, 18], and thus gender needs to be taken into account when investigating the association.

The overall aim of the current study was to estimate the prevalence of key sleep and circadian disorders (insomnia, DSWPD) and key sleep parameters (bed time, rise time, sleep-onset latency, total sleep time, time in bed and sleep efficiency) in adolescents within mental health care (clinical group) in comparison to a reference group from the general population adjusting for gender.

\section{Methods}

\section{Procedure}

This population-based study used data from the youth@ hordaland-survey of adolescents in Hordaland County in Western Norway. All adolescents born between 1993 and 1995 were invited in 2012 with an aim to reach all adolescents in late adolescence and high school age. The main aim of the survey was to assess the prevalence of mental health problems and service use in adolescents. Adolescents in upper secondary education received study information via e-mail, and one classroom school hour was allocated for completing the questionnaire. Those not in school received information by postal mail to their home address. The questionnaire was web-based. It covered a broad range of mental health issues, daily life functioning, use of health care and social services, and demographic characteristics, as well as a request for permission to obtain school data and link the information with national health registries. For those who consented to registry linkage, the data from the youth@ hordaland study were linked to the National Patient Registry (NPR), the official registry for specialist health care in Norway and provide data from specialized child and adolescent mental health care services. The linkage was performed by the registry owner at the Norwegian Directorate of Health and a deidentified dataset consisting of data from the NPR and the youth@ hordaland was used in the present study.

\section{Sample}

All adolescents born between 1993 and 1995 were invited $(N=19,430)$ to participate in the epidemiological study during the first months of 2012, of which 10,257 agreed, yielding a participation rate of $53 \%$. The validity of sleep data was assessed manually, with participants providing obvious invalid responses being omitted from further analyses. Invalid responses included 1) sleep-onset latency (SOL) or wake-after-sleep onset (WASO) more than $12 \mathrm{~h}$, 2) SOL + WASO longer than time in bed (TIB), and 3) negative values of sleep duration and sleep efficiency. The adolescents that consented to linkage to registries $(n=9411)$ and had valid sleep data are included in the present study $(n=9007)$.

\section{Representativeness}

In a study on an earlier linkage between NPR and youth@ hordaland study, those who did not consent to the linkage 
had more self-reported conduct problems, were somewhat older, and had a slightly increased rate of high-level alcohol consumption. However, the differences were all small in magnitude, with Cohen's $d$ effect sizes ranging from 0.09 to 0.26 [19].

\section{Instruments}

\section{Socio-demographics}

Gender and date of birth were identified through personal identity number in the Norwegian National Population Register. Exact age was estimated by calculating the interval of time between the date of birth and date of participation. Socioeconomic status (SES) was assessed both by perceived economic well-being and parental education. Perceived economic well-being was reported with three response options: "poorer than others", "equal to others", and "better than others." Maternal and paternal education were reported separately with three response options: "primary school", "secondary school", and "college or university".

\section{Sleep measures}

Insomnia was operationalized in alignment to the DSM-5 criteria [5]. Difficulties initiating and maintaining sleep (DIMS) were rated on a three-point Likert-scale with response options "not true", "somewhat true" and "certainly true". If confirmed (i.e., "somewhat true" or "certainly true"), adolescents were then asked how many days per week they experienced problems either initiating or maintaining sleep. Adolescents also provided information on the duration of DIMS. A joint question on tiredness/sleepiness was rated on a three-point Likert-scale with response options "not true", "somewhat true" and "certainly true". If confirmed, adolescents then reported the number of days per week they experienced sleepiness and tiredness, respectively. To meet the DSM-5 criteria for insomnia, adolescents had to report DIMS at least three times a week, with a duration of three months or more, as well as tiredness or sleepiness at least three days per week.

Delayed sleep-wake-phase disorder (DSWPD) was assessed by the following questions: "At what time do you usually go to bed?", "How much time does it take before you fall asleep (hours and minutes)?", "When do you usually get out of bed in the morning?", "How many nights per week do you have difficulties falling asleep (0-7)?", "How many nights per week do you have problems with nightly awakenings (0-7)?", "How often do you oversleep ("never", "seldom", "sometimes", "mostly", "always")?". The participants provided sleep data separately for weekdays and weekends. No information regarding the time frame of these symptoms was available. To establish a proxy for assessing DSWPD (as close as possible given the available sleep items) in line with the International Classification of Sleep Disorders-2 (American Academy of Sleep Medicine 2005), we employed the following criteria (as specified in Johnson et al. published in Pediatrics): (1) minimum 1-h shift in sleep-onset AND wake times from the weekdays to the weekend, (2) complaint of frequent ( $\geq 3$ days per week) difficulty falling asleep, (3) report of little or no ( $\leq 1$ day per week) difficulty maintaining sleep, and (4) frequent difficulty awakening (oversleep "sometimes" or more often).

Self-reported usual bedtime and rise time were indicated in hours and minutes using a scroll down menu; data were reported separately for weekdays and weekends. Time in bed (TIB) was calculated as the difference between bedtime and rise time. Sleep-onset latency (SOL) and wake-after-sleep onset (WASO) were indicated in hours and minutes, and sleep duration was defined as TIB minus SOL and WASO. Sleep efficiency was calculated as sleep duration divided by TIB multiplied by 100 (reported as a percentage; higher scores reflecting greater sleep efficiency). Sleep efficiency was analyzed as a continuous variable, with less than $85 \%$ as the cut-off for indications of poor sleep quality in accordance with Lacks and Morin [20]

\section{Psychiatric disorders}

Psychiatric disorders are based on Axis 1 diagnoses from the Norwegian Patient Registry (NPR). The psychiatric disorders were coded in accordance with Axis 1 in the ICD10 diagnostic manual by clinicians in the CAMHS, and we assigned all the diagnoses into broader diagnostic categories included in the present study: depression, anxiety, attentiondeficit/hyperactivity disorder (ADHD), conduct disorder, trauma-related disorder, autism, eating disorder, psychotic disorders. Sleep diagnoses (F510 Non-organic insomnia and non- organic disorder of the sleep-wake cycle) were also included. A range of less common psychiatric diagnoses were categorized as 'other psychiatric diagnoses' but was not used in these analyses due to a large conceptual heterogeneity [19].

The timing of the contact with CAMHS was categorized into contact initiated before, at the same time and after the youth@hordaland.

\section{Ethics}

The study was approved by the Regional Committee for Medical and Health Research Ethics (REC) in Western Norway (2011/811/REK Vest) and NSD (371,974 and 259,631).

Following the regulations from the REC and Norwegian health authorities, adolescents aged 16 years and older can make decisions regarding their health (including participation in health studies), and thus gave consent themselves 
to participate in the current study and for the linkage to registries. Parents/guardians have the right to be informed, and in the current study, and all parents/guardians received information about the study in advance.

\section{Statistical analyses}

Independent samples $t$-tests and chi-squared tests were used to examine differences in demographic variables for the adolescents in the clinical sample, i.e. those within the CAMHS (i.e. the clinical group) and in the reference group (i.e. those not in contact with CAMHS). The various psychiatric diagnosis in the clinical group was all compared to the reference group on insomnia and DSWPD using log-link binomial regression analysis. Being similar to how risk ratios (RR) are calculated, the estimates are presented as prevalence ratios (PR) given the cross-sectional nature of this study. Similarly, the sleep parameter in adolescents across diagnosis in the clinical group was compared to adolescents in the reference group by computing estimated marginal means (EMM), adjusting for gender.

\section{Results}

\section{Characteristics of the sample}

The total sample $(n=9077)$ consisted of $53.4 \%$ girls, and the mean age was 17.4 years (SD 0.83), range 16-19 years. There were significantly more girls than boys in the clinical group compared to the reference group, while no significant age difference was observed (see Table 1 for details).

In all, 911 participants (10\%) in youth@hordaland had been in contact with CAMHS and comprised the clinical group. Among these, two-thirds $(66.4 \%)$ had been in contact before the onset of this epidemiological health survey, $24.8 \%$ were in contact during the data collection period, while $8.8 \%$ initiated the contact after the health survey. Among the participants in the clinical group, only six adolescents were registered as having a sleep disorder diagnosis $(0.6 \%$ of people connected with services).

\section{Insomnia and delayed sleep in adolescents with psychiatric disorders compared to a reference group}

Prevalence and PR for insomnia and DSWPD for adolescents with a psychiatric diagnosis in the clinical sample, in comparison to the reference group, are presented in Table 2. In the reference group, $16.7 \%$ presented with insomnia, while in the clinical group, the prevalence ranged from $28.7 \%$ for ADHD (PR: 1.79, CI 95\% [1.41-2.29]) to $48 \%$ for depression (PR: 2.53, CI 95\% [2.19-2.92]). DSPS was present for $2.9 \%$ of the reference group, while the prevalence among those with psychiatric disorders ranged from $0 \%$ for eating disorders to $20 \%$ for the conduct disorder group (PR: 7.28, CI 95\% [3.52-15.05]).

\section{Sleep parameters among adolescents with psychiatric disorders compared to a reference group}

Estimates of sleep duration, SOL, WASO, and sleep efficiency (adjusting for gender) are displayed in Fig. 1. Compared to the reference group, adolescents diagnosed with depression, ADHD, conduct disorder (ps $<0.001$ ), trauma and eating disorder $(p<0.05)$ had significantly shorter sleep duration. SOL was significantly longer among adolescents with depression, anxiety, ADHD, conduct disorder, trauma, eating disorder, as well as autism $(p<0.001)$ compared to the reference group. With regard to WASO, adolescents with depression, anxiety, ADHD, eating disorder, conduct disorder ( $\mathrm{ps}<0.001)$, and psychosis and trauma $(p<0.05)$ had significantly longer WASO compared to the reference group. Finally, the estimated sleep efficiency was significantly lower for all diagnostic groups, except for psychosis (see Fig. 1 for details).
Table 1 Demographic characteristic of the linked youth@hordaland and the Norwegian Patient Registry (NPR)-dataset and separately for those that have and have not been in contact with CAMHS

\begin{tabular}{llll}
\hline & Total (9077) & CAMHS contact (911) & Non-contact (8166) \\
\hline Girls \% $(n)$ & $53.4 \%$ & $60.3 \%(549)$ & $52.7 \%(4301)$ \\
Age in years, mean (SD) & $17.4(.83)$ & $17.3(.83)$ & $17.4(.83)$ \\
Highest education in the family & & & \\
Primary & $4.1 \%(364)$ & $6.1 \%(55)$ & $3.8 \%(309)$ \\
Secondary & $31 \%(268)$ & $29.8 \%(268)$ & $31,1 \%(2510)$ \\
College/university & $46.0 \%(4113)$ & $37.8 \%(339)$ & $46.9 \%(3774)$ \\
Don't know & $18.9 \%(1695)$ & $26.4 \%(237)$ & $18.1 \%(1458)$ \\
Insomnia & $19.2 \%(1980)$ & $34.4 \%(329)$ & $17.7 \%(1651)$ \\
Delayed sleep phase & $1.9 \%(175)$ & $4.0 \%(37)$ & $2.1 \%(212)$ \\
\hline
\end{tabular}


Table 2 Prevalence of insomnia and delayed sleep phase and the prevalence ratios $(\mathrm{PR})$ according to psychiatric diagnosis

\begin{tabular}{|c|c|c|c|c|c|c|}
\hline \multirow[t]{2}{*}{ Diagnosis from the NPR } & \multicolumn{3}{|c|}{$\begin{array}{l}\text { Insomnia DSM-5 from the youth@ } \\
\text { hordaland }\end{array}$} & \multicolumn{3}{|c|}{$\begin{array}{l}\text { Delayed sleep-wake-phase syn- } \\
\text { drome (DSWPD)from the Youth@ } \\
\text { hordaland }\end{array}$} \\
\hline & $\%$ & $\mathrm{PR}^{*}$ & $95 \% \mathrm{CI}$ & $\%$ & PR & $95 \% \mathrm{CI}$ \\
\hline $\begin{array}{l}\text { Not in contact with CAMHS } \\
\text { (ref) }(n=8166) \mathrm{s}\end{array}$ & $16.7 \%$ & - & - & $2.9 \%$ & - & - \\
\hline Depression $(n=221)$ & $48.0 \%$ & 2.53 & $2.19-2.92$ & $7.4 \%$ & 2.38 & $1.46-3.89$ \\
\hline Anxiety $(n=167)$ & $31.7 \%$ & 1.73 & $1.39-2.17$ & $5.6 \%$ & 1.82 & $0.95-3.49$ \\
\hline $\operatorname{ADHD}(n=160)$ & $28.7 \%$ & 1.79 & $1.41-2.29$ & $9.0 \%$ & 3.16 & $1.89-5.29$ \\
\hline Conduct $(n=30)$ & $40.0 \%$ & 2.64 & $1.74-3.99$ & $20.0 \%$ & 7.28 & $3.52-15.05$ \\
\hline Trauma $(n=77)$ & $40.3 \%$ & 2.14 & $1.64-2.80$ & $9.2 \%$ & 2.94 & $1.44-6.01$ \\
\hline Psychosis $(n=22)$ & $45.5 \%$ & 2.33 & $1.49-3.67$ & $9.1 \%$ & $* *$ & $* *$ \\
\hline Autism $(n=51)$ & $29.4 \%$ & 2.07 & $1.37-3.14$ & $6.0 \%$ & $* *$ & $* *$ \\
\hline Eating disorder $(n=56)$ & $42.9 \%$ & 2.05 & $1.51-2.79$ & $0 \%$ & $* *$ & $* *$ \\
\hline
\end{tabular}

*Adjusted for gender

**Analyses were not performed when cells had fewer than five cases

\section{Discussion}

The present registry-linked population-based study of older adolescents provides support for sleep problems as a transdiagnostic problem across psychiatric disorders. Sleep disorders that were diagnosed within CAMHS were very rare, with only $0.6 \%$ of the adolescents having received a formal sleep disorder diagnosis. However, adolescents with diagnosed psychiatric disorders had significantly higher prevalence of insomnia and DSWPD, and overall most diagnostic groups also had substantially shorter sleep duration, lower sleep efficiency, as well as longer SOL and WASO than the reference group.

Insomnia is highly prevalent both in adolescence [1], and the general adult population [21]. The present study confirms even higher prevalence for adolescents with a psychiatric disorder, with all diagnostic groups presenting with an increased risk. Sleep problems are included as symptoms for a range of psychiatric disorders listed in the DSM-5, and there are also likely common underlying mechanisms, including biological, psychological, and social factors [22]. For instance, for depression, which was the disorder with the highest prevalence of insomnia (48\%), these findings correspond well with the established association between depression and sleep problems in adolescence [23].

DSWPD is a much less prevalent condition in the general population, but peaks in late adolescence [24]. The current study provides new evidence of even higher prevalence of DSWPD among adolescents with psychiatric disorders. While the prevalence of DSWPD was low in the reference group at $2.9 \%$, the prevalence among those diagnosed with a psychiatric disorder was higher, with a disorder-specific pattern. Specifically, the highest prevalences were observed among adolescents with conduct disorders at $20 \%$, while there were no identified cases among adolescents with eating disorders. The strong association between DSWPD and conduct disorder and mood disorders is also in line with the pattern of observed oversleeping on weekends among adolescents with these disorders in a US population-based study [4]. Due to the low prevalence, and the related lack of statistical power, we did not perform statistical analysis for the less prevalent psychiatric diagnosis.

Suboptimal sleep patterns and poor sleep quality in general were present across all psychiatric disorders. In particular, short sleep duration caused by long SOL and WASO, was evident. These findings are consistent with the observed short sleep duration during weekdays across diagnostic categories in the above-mentioned US population-based study by Zhang et al. [4]. The clinical significance of the short sleep duration was underscored by the low sleep efficiencies across most diagnostic groups, well below the established clinical cut-off of $85 \%$.

It is noteworthy that the high prevalence of sleep problems as indicated by questionnaires in the epidemiological study was not mirrored by registered diagnosis of sleep disorders in the official registries. The low prevalence of diagnosed sleep problems within CAMHS may not be that surprising, given a similar low prevalence of diagnosed sleep disorders among adults receiving mental health services in Norway [25]. The low prevalence of diagnosed disorders might reflect that sleep assessment and treatment is not integrated into clinical practice, or that while acknowledged, sleep problems are not formalized in patient journals, nor are they seen as severe enough to warrant a diagnosis.

The high prevalence of sleep problems across diagnostic categories supports the central role of sleep problems in psychiatric disorders, which is also central from a transdiagnostic view [16]. Although the present study precludes 

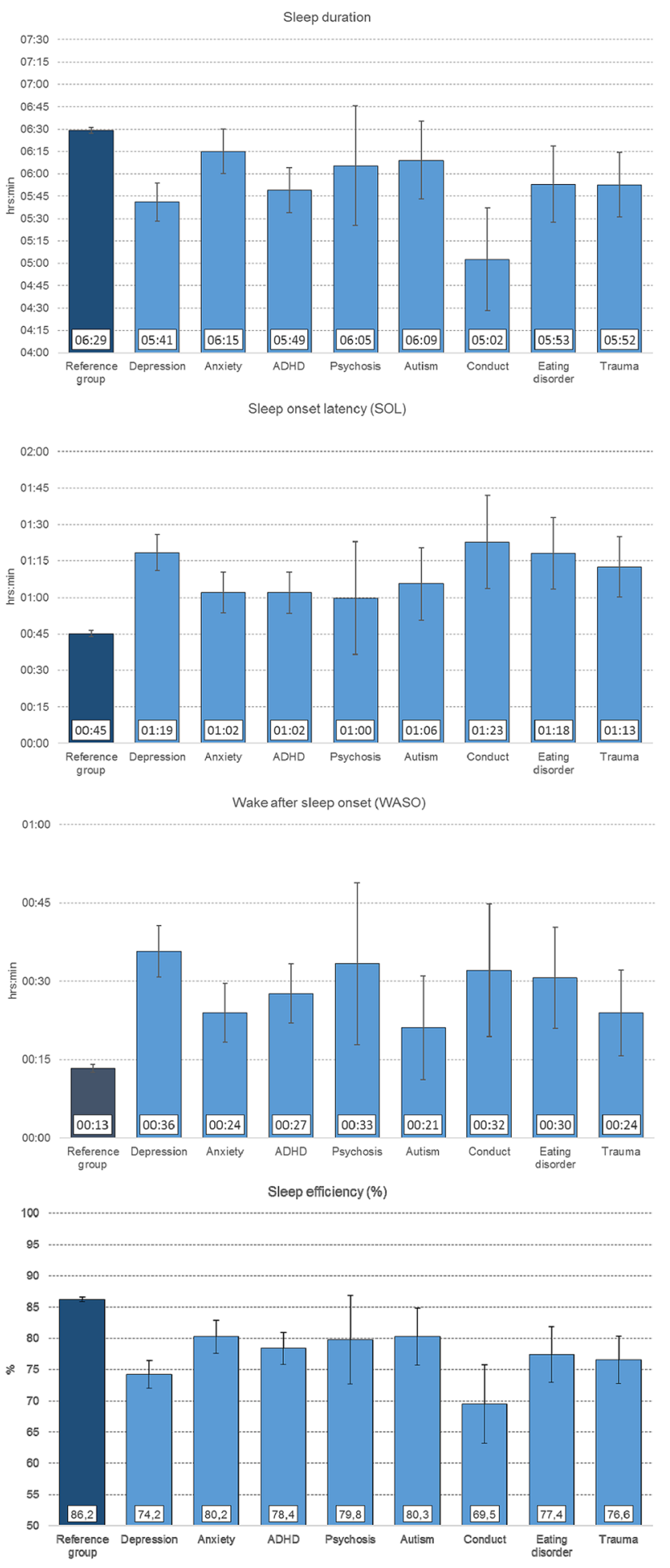

Fig. 1 Sleep duration, sleep-onset latency, wake-after-sleep onset and sleep efficiency for adolescents with a diagnosed disorder of depression, anxiety, ADHD, psychotic disorders, autism, conduct, eating disorder and trauma in comparison to a reference group investigating possible mechanisms involved in these associations, previous studies and models indicate that they share common underlying processes, for instance common genetic influences, biological processes as well as psychological processes $[16,22,26]$. Future studies are needed to understand the mechanism, for instance the strong co-occurrence of DSWPD among adolescents with conduct disorder should be investigated to further elucidate the etiology and pathophysiology.

Some methodological considerations should be noted. The strength of the study is the heterogeneous patient group and clinically diagnosed psychiatric disorders. However, this is was also a weakness of the study, since the clinical assessment may vary across clinicians and is not standardized for the present study. Further, the reference group may include people with mental illness who have not been connected with treatment services. Some of the diagnostic groups were small, such as psychotic disorders, which limits statistical power and the conclusions that can be drawn. The overlap between insomnia and DSWPD was not specifically addressed in the current study, although the overlap is often high [24] and this has important clinical implications. Further, only problems initiating and maintaining sleep were included, and early morning awakenings were not specifically addressed, which is a limitation of the study. Another limitation of the insomnia operalisation is that functional impact was addressed by sleepiness and tiredness, and the perceived impact on other domains was not included. We cannot comment on temporality between the sleep and the psychiatric disorders. However, previous studies have suggested that the link between sleep and specific psychiatric disorders is likely to be bidirectional, with sleep both predicting the development of mental health problems, and impacting treatment [27]. Also, one cannot disregard the possibility that there may be diagnosis-specific directionality, where some diagnoses are bidirectionally associated with sleep, while others have a unidirectional association [7].

\section{Conclusion}

These results confirm the transdiagnostic role of sleep problems in mental disorders in adolescence, emphasizing the importance of thorough sleep assessments which should encompass general sleep patterns and disorders like insomnia and DSWPD. These findings also point to the importance of preventive interventions that could improve sleep among adolescents and might even be one pathway to prevent the development of mental health problems. Finally, interventions for sleep and circadian functioning in youth must become widely available [28]. Tailored transdiagnostic sleep interventions for adolescents that aim at improving 
sleep and circadian rhythm are good examples of treatments that might be included in the CAMHS [29]. In addition to the expected improvement in sleep problems, they may also give additional benefit for the symptoms of the psychiatric disorders [30, 31].

Acknowledgement Open Access funding provided by University of Bergen. We want to thank the Bergen Child Study group and most importantly we are grateful to all participants that made this study possible

\section{Compliance with ethical standards}

Conflicts of interest The authors declare that they have no conflicts of interest.

Ethics approval All procedures in the present study were in accordance with the ethical standards of the institutional and/or national research committee and with the 1964 Helsinki Declaration and its later amendments or comparable ethical standards. The study was approved by the Regional Committee for Medical and Health Research Ethics (REC) in Western Norway (2011/811/REK Vest) and NSD (371974 and 259631).

Consent to participate Following the regulations from the REC and Norwegian health authorities, adolescents aged 16 years and older can make decisions regarding their health (including participation in health studies), and thus gave consent themselves to participate in the current study and for the linkage to registries. Parents/guardians have the right to be informed, and in the current study, and all parents/guardians received information about the study in advance.

Open Access This article is licensed under a Creative Commons Attribution 4.0 International License, which permits use, sharing, adaptation, distribution and reproduction in any medium or format, as long as you give appropriate credit to the original author(s) and the source, provide a link to the Creative Commons licence, and indicate if changes were made. The images or other third party material in this article are included in the article's Creative Commons licence, unless indicated otherwise in a credit line to the material. If material is not included in the article's Creative Commons licence and your intended use is not permitted by statutory regulation or exceeds the permitted use, you will need to obtain permission directly from the copyright holder. To view a copy of this licence, visit http://creativecommons.org/licenses/by/4.0/.

\section{Appendix}

\section{See Table 3.}

Table 3 Operationalization of broader diagnostic categories in the linked youth@ @ordaland-NPR sample

\begin{tabular}{|c|c|c|}
\hline Diagnostic category & ICD-10 code & ICD-10 diagnosis \\
\hline \multirow[t]{21}{*}{ Anxiety disorders } & F400 & Agoraphobia \\
\hline & F4000 & Agoraphobia \\
\hline & F401 & Social phobia \\
\hline & F402 & Specific (isolated) phobias \\
\hline & F408 & Other phobic anxiety disorders \\
\hline & F409 & Phobic anxiety disorder, unspecified \\
\hline & F410 & Panic disorder [episodic paroxysmal anxiety] \\
\hline & F411 & Generalized anxiety disorder \\
\hline & F412 & Mixed anxiety and depressive disorder \\
\hline & F413 & Other mixed anxiety disorders \\
\hline & F418 & Other specified anxiety disorders \\
\hline & F419 & Anxiety disorder, unspecified \\
\hline & F420 & Predominantly obsessional thoughts or ruminations \\
\hline & F421 & Predominantly compulsive acts [obsessional rituals] \\
\hline & F422 & Mixed obsessional thoughts and acts \\
\hline & F429 & Obsessive-compulsive disorder, unspecified \\
\hline & F452 & Hypochondriacal disorders \\
\hline & F930 & Separation anxiety disorder of childhood \\
\hline & F931 & Phobic anxiety disorder of childhood \\
\hline & F932 & Social anxiety disorder of childhood \\
\hline & F940 & Elective mutism \\
\hline \multirow[t]{6}{*}{ Mood disorders } & F310 & Bipolar affective disorder, current episode hypomanic \\
\hline & F311 & Bipolar affective disorder, current episode manic without psychotic symptoms \\
\hline & F312 & Bipolar disorder, current episode manic severe with psychotic features \\
\hline & F313 & Bipolar affective disorder, current episode mild or moderate depression \\
\hline & F314 & Bipolar disorder, current episode depressed, severe, without psychotic features \\
\hline & F316 & Bipolar affective disorder, current episode mixed \\
\hline
\end{tabular}


Table 3 (continued)

\begin{tabular}{|c|c|c|}
\hline Diagnostic category & ICD-10 code & ICD-10 diagnosis \\
\hline & F317 & Bipolar affective disorder, currently in remission \\
\hline & F318 & Other bipolar disorders \\
\hline & F319 & Bipolar affective disorder, unspecified \\
\hline & F320 & Mild depressive episode \\
\hline & F3200 & Mild depressive episode without somatic syndrome \\
\hline & F3201 & Mild depressive episode with somatic syndrome \\
\hline & F321 & Moderate depressive episode \\
\hline & F322 & Severe depressive episode without psychotic symptoms \\
\hline & F323 & Major depressive disorder, single episode, severe with psychotic features \\
\hline & F328 & Other depressive episodes \\
\hline & F329 & Depressive episode, unspecified \\
\hline & F330 & Major depressive disorder, recurrent, mild \\
\hline & F331 & Recurrent depressive disorder, current episode moderate \\
\hline & F3310 & Recurrent depressive disorder, current episode moderate, without somatic syndrome \\
\hline & F332 & Recurrent depressive disorder, current episode severe without psychotic symptoms \\
\hline & F333 & Recurrent depressive disorder, current episode severe with psychotic symptoms \\
\hline & F338 & Other recurrent depressive disorders \\
\hline & F339 & Major depressive disorder, recurrent, unspecified \\
\hline & F341 & Dysthymic disorder \\
\hline & F348 & Other persistent mood [affective] disorders \\
\hline & F349 & Persistent mood [affective] disorder, unspecified \\
\hline & F381 & Other recurrent mood [affective] disorders \\
\hline & F412 & Mixed anxiety and depressive disorder \\
\hline \multirow[t]{10}{*}{ Trauma-related disorders } & F430 & Acute stress reaction \\
\hline & F431 & Post-traumatic stress disorder \\
\hline & F4320 & Adjustment disorder, unspecified \\
\hline & F4321 & Adjustment disorder with depressed mood \\
\hline & $\mathrm{F} 4322$ & Adjustment disorder with anxiety \\
\hline & $\mathrm{F} 4323$ & Adjustment disorder with mixed anxiety and depressed mood \\
\hline & $\mathrm{F} 4324$ & Adjustment disorder with disturbance of conduct \\
\hline & F4325 & Adjustment disorder with mixed disturbance of emotions and conduct \\
\hline & F438 & Other reactions to severe stress \\
\hline & F439 & Reaction to severe stress, unspecified \\
\hline \multirow[t]{6}{*}{ Eating disorders } & F500 & Anorexia nervosa \\
\hline & F501 & Atypical anorexia nervosa \\
\hline & F503 & Atypical bulimia nervosa \\
\hline & F504 & Overeating associated with other psychological disturbances \\
\hline & F505 & Vomiting associated with other psychological disturbances \\
\hline & F509 & Eating disorder, unspecified \\
\hline \multirow[t]{5}{*}{ Autism-spectrum disorders (ASD) } & F840 & Childhood autism \\
\hline & F841 & Atypical autism \\
\hline & F845 & Asperger syndrome \\
\hline & F848 & Other pervasive developmental disorders \\
\hline & F849 & Pervasive developmental disorder, unspecified \\
\hline \multirow[t]{6}{*}{ Conduct disorders } & F910 & Conduct disorder confined to family context \\
\hline & F911 & Unsocialized conduct disorder \\
\hline & F912 & Conduct disorder, adolescent-onset type \\
\hline & F913 & Oppositional defiant disorder \\
\hline & F918 & Other conduct disorders \\
\hline & F919 & Conduct disorder, unspecified \\
\hline
\end{tabular}


Table 3 (continued)

\begin{tabular}{|c|c|c|}
\hline Diagnostic category & ICD-10 code & ICD-10 diagnosis \\
\hline & F920 & Depressive conduct disorder \\
\hline & F928 & Other mixed disorders of conduct and emotions \\
\hline & F929 & Mixed disorder of conduct and emotions, unspecified \\
\hline \multirow{4}{*}{$\begin{array}{l}\text { Attention-deficit/hyperactivity } \\
\text { disorder (ADHD) }\end{array}$} & F900 & Hyperkinetic disorders \\
\hline & F901 & Disturbance of activity and attention \\
\hline & F908 & Other hyperkinetic disorders \\
\hline & F909 & Attention-deficit hyperactivity disorder, unspecified type \\
\hline \multirow[t]{14}{*}{ Psychotic disorders } & F203 & Undifferentiated schizophrenia \\
\hline & F208 & Other schizophrenia \\
\hline & F209 & Schizophrenia, unspecified \\
\hline & F2090 & Schizophrenia, unspecified \\
\hline & $\mathrm{F} 21$ & Schizotypal disorder \\
\hline & F231 & Acute polymorphic psychotic disorder with symptoms of schizophrenia \\
\hline & F233 & Acute paranoid psychosis without acute stressor \\
\hline & F239 & Acute and transient psychotic disorder, unspecified \\
\hline & F2390 & Acute and transient psychotic disorder, unspecified \\
\hline & F28 & Other psychotic disorder not due to a substance or known physiological condition \\
\hline & $\mathrm{F} 29$ & Unspecified nonorganic psychosis \\
\hline & F312 & Bipolar disorder, current episode manic severe with psychotic features \\
\hline & F323 & Major depressive disorder, single episode, severe with psychotic features \\
\hline & F333 & Recurrent depressive disorder, current episode severe with psychotic symptoms \\
\hline \multirow[t]{25}{*}{ Other axis 1 psychiatric diagnoses } & F448 & Other dissociative and conversion disorders \\
\hline & F449 & Dissociative [conversion] disorder, unspecified \\
\hline & F454 & Persistent somatoform pain disorder \\
\hline & F458 & Other somatoform disorders \\
\hline & F480 & Neurasthenia \\
\hline & F489 & Neurotic disorder, unspecified \\
\hline & F510 & Nonorganic insomnia \\
\hline & F512 & Nonorganic disorder of the sleep-wake schedule \\
\hline & F54 & $\begin{array}{l}\text { Psychological and behavioral factors associated with disorders or diseases classified else- } \\
\text { where }\end{array}$ \\
\hline & F601 & Schizoid personality disorder \\
\hline & F633 & Trichotillomania \\
\hline & F640 & Transsexualism \\
\hline & F659 & Disorder of sexual preference, unspecified \\
\hline & F933 & Sibling rivalry disorder \\
\hline & F938 & Other childhood emotional disorders \\
\hline & F939 & Childhood emotional disorder, unspecified \\
\hline & F941 & Reactive attachment disorder of childhood \\
\hline & F942 & Disinhibited attachment disorder of childhood \\
\hline & F948 & Other childhood disorders of social functioning \\
\hline & F950 & Transient tic disorder \\
\hline & F951 & Chronic motor or vocal tic disorder \\
\hline & F952 & Combined vocal and multiple motor tic disorder [de la Tourette] \\
\hline & F980 & Nonorganic enuresis \\
\hline & F981 & Nonorganic encopresis \\
\hline & F988 & $\begin{array}{l}\text { Other specified behavioral and emotional disorders with onset usually occurring in childhood } \\
\text { and adolescence }\end{array}$ \\
\hline
\end{tabular}


Table 3 (continued)

\begin{tabular}{|c|c|c|}
\hline Diagnostic category & ICD-10 code & ICD-10 diagnosis \\
\hline & F989 & $\begin{array}{l}\text { Unspecified behavioral and emotional disorders with onset usually occurring in childhood } \\
\text { and adolescence }\end{array}$ \\
\hline & F99 & Mental disorder, not otherwise specified \\
\hline & $\mathrm{R} 418$ & Other symptoms and signs involving cognitive functions and awareness \\
\hline & $\mathrm{R} 440$ & Auditory hallucinations \\
\hline & R441 & Visual hallucinations \\
\hline & R452 & Unhappiness \\
\hline & R454 & Irritability and anger \\
\hline & R455 & Hostility \\
\hline & R457 & State of emotional shock and stress, unspecified \\
\hline & R458 & Other symptoms and signs involving emotional state \\
\hline & R466 & Undue concern and preoccupation with stressful events \\
\hline
\end{tabular}

\section{References}

1. Hysing $\mathrm{M}$ et al (2013) Sleep patterns and insomnia among adolescents: a population-based study. J Sleep Res 22:549-556

2. Maslowsky J, Ozer EJ (2014) Developmental trends in sleep duration in adolescence and young adulthood: evidence from a national United States sample. J Adolesc Health 54(6):691-697

3. Johnson EO et al (2006) Epidemiology of DSM-IV insomnia in adolescence: lifetime prevalence, chronicity, and an emergent gender difference. Pediatrics 117(2):e247-e256

4. Zhang J et al (2017) Sleep patterns and mental health correlates in US adolescents. J Pediatr 182:137-143

5. American Psychiatric Association (2013) Diagnostic and statistical manual of mental disorders, 5th edn. American Psychiatric Association, Arlington, VA

6. Baglioni $\mathrm{C}$ et al (2011) Insomnia as a predictor of depression: a meta-analytic evaluation of longitudinal epidemiological studies. J Affect Disord 135(1-3):10-19

7. Alvaro PK et al (2017) The direction of the relationship between symptoms of insomnia and psychiatric disorders in adolescents. J Affect Disord 207:167-174

8. American Psychological Association (2000) Diagnostic and statistical manual of mental disorders, 4th edn. American Psychological Association, Washington DC

9. Wakefield JC (2012) DSM-5: proposed changes to depressive disorders. Curr Med Res Opin 28(3):335-343

10. Lichstein KL (2006) Secondary insomnia: a myth dismissed. Sleep Med Rev 10(1):3-5

11. Reigstad B et al (2010) Prevalences and correlates of sleep problems among adolescents in specialty mental health services and in the community: what differs? Nord J Psychiatry 64(3):172-180

12. Kallestad $\mathrm{H}$ et al (2012) Impact of sleep disturbance on patients in treatment for mental disorders. BMC Psychiatry 12:179

13. Gau SS, Chiang HL (2009) Sleep problems and disorders among adolescents with persistent and subthreshold attention-deficit/ hyperactivity disorders. Sleep 32(5):671-679

14. Blank $\mathrm{M}$ et al (2015) Health correlates of insomnia symptoms and comorbid mental disorders in a nationally representative sample of US adolescents. Sleep 38(2):197-204

15. Roberts RE, Duong HT (2017) Is there an association between short sleep duration and adolescent anxiety disorders? Sleep Med 30:82-87

16. Harvey AG et al (2011) Sleep disturbance as transdiagnostic: consideration of neurobiological mechanisms. Clin Psychol Rev 31(2):225-235
17. Merikangas KR et al (2010) Lifetime prevalence of mental disorders in U.S. adolescents results from the National Comorbidity Survey Replication-Adolescent Supplement (NCS-A). J Am Acad Child Adolesc Psychiatry 49(10): 980-989

18. Georgiades K et al (2019) Six-month prevalence of mental disorders and service contacts among children and youth in Ontario: evidence from the 2014 Ontario child health study. Can J Psychiatry 64(4):246-255

19. Heradstveit $\mathrm{O}$ et al (2019) Psychiatric diagnoses differ considerably in their associations with alcohol/drug-related problems among adolescents. A Norwegian population-based survey linked with national patient registry data. Front Psychol 10:1003

20. Lacks P, Morin CM (1992) Recent advances in the assessment and treatment of insomnia. J Consult Clin Psychol 60(4):586-594

21. Sivertsen B et al. (2020) Sleep patterns and insomnia in a large population-based study of middle-aged and older adults: the Tromso study 2015-2016. J Sleep Res. https://doi.org/10.1111/ jsr.13095

22. Blake MJ, Trinder JA, Allen NB (2018) Mechanisms underlying the association between insomnia, anxiety, and depression in adolescence: Implications for behavioral sleep interventions. Clin Psychol Rev 63:25-40

23. Lovato N, Gradisar M (2014) A meta-analysis and model of the relationship between sleep and depression in adolescents: recommendations for future research and clinical practice. Sleep Med Rev 18(6):521-529

24. Sivertsen B et al (2013) Delayed sleep phase syndrome in adolescents: prevalence and correlates in a large population based study. BMC Public Health 13:1163

25. Kallestad H et al (2011) Differences between patients' and clinicians' report of sleep disturbance: a field study in mental health care in Norway. BMC Psychiatry 11:186

26. Goldstein AN, Walker MP (2014) The role of sleep in emotional brain function. Annu Rev Clin Psychol 10:679-708

27. Kleim B et al (2016) Effects of sleep after experimental trauma on intrusive emotional memories. Sleep 39(12):2125-2132

28. Blake MJ et al (2017) Systematic review and meta-analysis of adolescent cognitive-behavioral sleep interventions. Clin Child Fam Psychol Rev 20(3):227-249

29. Dong L et al (2020) A transdiagnostic sleep and circadian intervention for adolescents: six-month follow-up of a randomized controlled trial. J Child Psychol Psychiatry 61(6):653-661

30. Cremone-Caira A et al (2020) Effects of sleep extension on inhibitory control in children with aDHD: a pilot study. J Atten Disord 24(4):601-610 
31. Blake M et al (2016) The SENSE study: post intervention effects of a randomized controlled trial of a cognitive-behavioral and mindfulness-based group sleep improvement intervention among at-risk adolescents. J Consult Clin Psychol 84(12):1039-1051 